\title{
Epidemiologia e diferenças regionais da retinopatia diabética em Pernambuco, Brasil
}

\author{
Epidemiology and regional differences of diabetic retinopathy in \\ Pernambuco, Brazil
}

Paulo Henrique Gonçalves Escarião ${ }^{1}$

Tiago Eug'ênio Faria de Arantes ${ }^{2}$

Natanael Cavalcanti Figueiroa Filho ${ }^{3}$

Ricardo de Deus Urtiga ${ }^{4}$

Telma Lúcia Tabosa Florêncio ${ }^{5}$

Ana Lúcia de Andrade Lima Arcoverde ${ }^{6}$
Trabalho realizado no Departamento de Oftalmologia da Fundação Altino Ventura. Recife (PE) - Brasil.

Preceptor de Retina e Vítreo da Fundação Altino Ventura e Hospital de Olhos de Pernambuco; Coordenador do Projeto para Prevenção da Cegueira por Retinopatia Diabética da Fundação Altino Ventura, Recife (PE) Brasil.

2 Pós-Graduando do Setor de Uveítes e AIDS do Departamento de Oftalmologia da Escola Paulista de Medicina da Universidade Federal de São Paulo - UNIFESP São Paulo (SP) - Brasil.

Oftalmologista pela Residência da Fundação Altino Ventura, Recife (PE) - Brasil.

${ }^{4}$ Oftalmologista pela Residência da Fundação Altino Ventura. Recife (PE) - Brasil.

${ }_{5}^{5}$ Preceptor de Retina e Vítreo da Fundação Altino Ventura e Hospital de Olhos de Pernambuco. Recife (PE) Brasil.

${ }^{6}$ Preceptor de Retina e Vítreo da Fundação Altino Ventura e Hospital de Olhos de Pernambuco. Recife (PE) Brasil.

Endereço para correspondência: Paulo Escarião. Fundação Altino Ventura - Departamento de Retina e Vítreo - Recife (PE) - Brasil. Rua da Soledade, 170 - Recife (PE) CEP 50040-070

Email: phescariao@hotmail.com

Recebido para publicação em 11.02.2006

Versão recebida em 22.11.2007

Aprovação em 06.12.2007

Nota Editorial: Depois de concluída a análise do artigo sob sigilo editorial e com a anuência da Dra. Luciene Chaves Fernandes sobre a divulgação de seu nome como revisora, agradecemos sua participação neste processo.

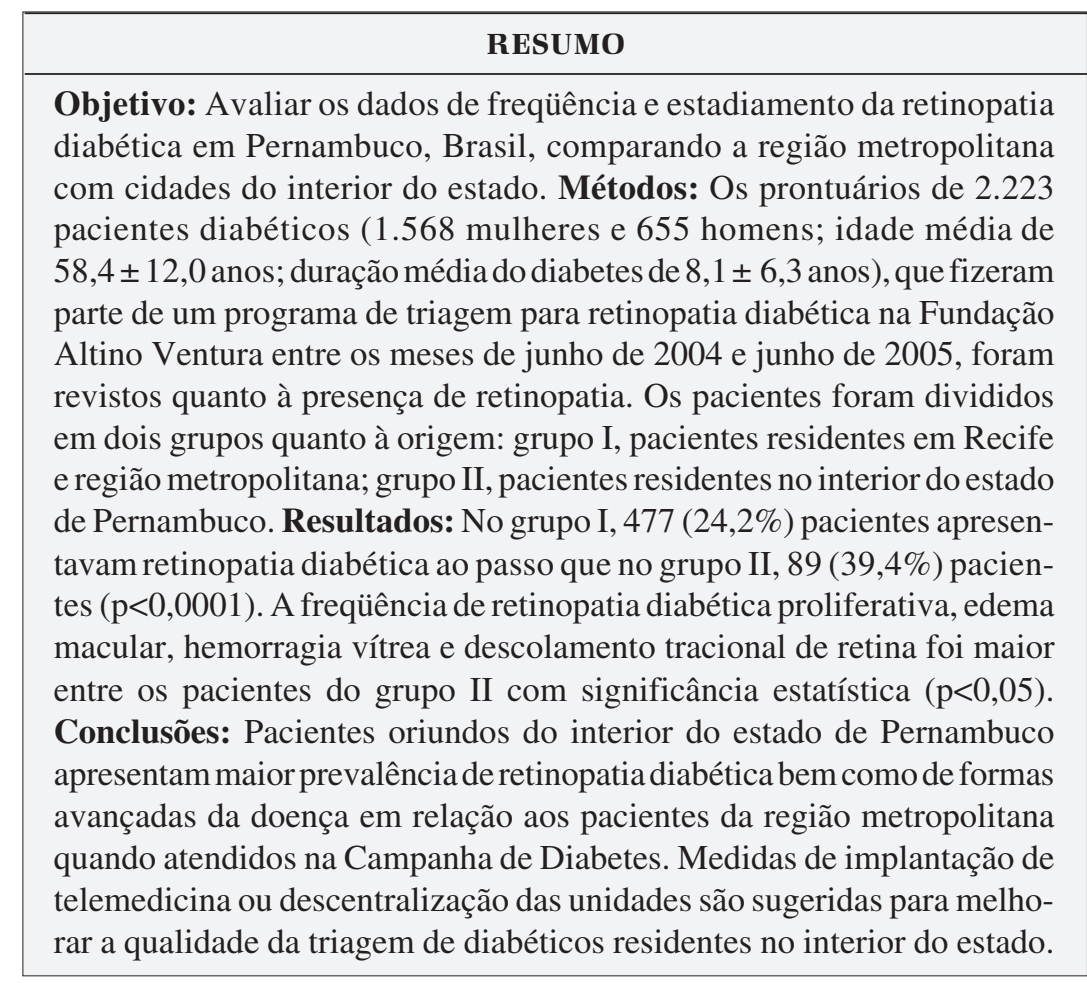

Descritores: Retinopatia diabética/etiologia; Retinopatia diabética/diagnóstico; Prevalência; Triagem

\section{INTRODUÇÃO}

As complicações microvasculares do diabetes mellitus na retina constituem-se na principal causa de cegueira da população economicamente ativa no Brasil ${ }^{(1)}$ e no mundo ${ }^{(2-3)}$. A prevenção baseia-se no controle clínico rigoroso e detecção precoce de alterações fundoscópicas que ameacem a visão, como edema macular diabético e neovascularização retiniana ${ }^{(4-5)}$. O tratamento dessas alterações é eficaz na prevenção da cegueira quando instituído precocemente ${ }^{(5)}$.

A realização de campanhas de saúde pública com o intuito de detecção precoce dessas alterações por meio de triagem visual periódica e inclusão do paciente num programa de acompanhamento sistemático visa reduzir a prevalência de cegueira, bem como, custos com o tratamento e reabilitação de deficientes visuais ${ }^{(6-8)}$.

Um programa de controle ideal para detecção da retinopatia deve apre- 
sentar ampla cobertura sobre a população de diabéticos contida no programa. Pouco é descrito sobre diferenças entre prevalência e estadiamento da retinopatia entre diabéticos residentes em uma área metropolitana em relação a diabéticos residentes em áreas rurais ou cidades do interior com condições socioeconômicas mais precárias, particularmente no nordeste do Brasil. Além de possíveis dificuldades do acesso desses pacientes a um programa de triagem.

O objetivo desse estudo foi avaliar dados de prevalência e estadiamento da retinopatia diabética em Pernambuco, Brasil, comparando a região metropolitana com cidades do interior do estado.

\section{MÉTODOS}

Foi realizado estudo retrospectivo na Fundação Altino Ventura em Recife, Pernambuco, Brasil no período de junho de 2004 a junho de 2005, entre pacientes que participaram da Campanha de Prevenção à Cegueira por Diabetes. Foram examinados 2.223 diabéticos (1.568 mulheres e 655 homens; média de idade de 59,3 $\pm 12,0$ anos), sendo excluídos 22 pacientes por apresentarem catarata que impossibilitava a avaliação da retina.

Durante o atendimento, os pacientes eram questionados quanto ao tipo de tratamento (dieta, hipoglicemiante oral ou insulina), tempo de diagnóstico e exame fundoscópico prévio.

A retinopatia diabética $(\mathrm{RD})$ foi avaliada através de oftalmoscopia binocular indireta e biomicroscopia de fundo, sob midríase e realizada por médico especialista em retina. $\mathrm{O}$ estadiamento, baseado em critérios do $\operatorname{ETDRS}^{(9)}$, foi dividido em: forma nãoproliferativa (RDNP) leve (presença de microaneurismas e/ou hemorragias intra-retinianas em menos de 4 quadrantes), moderada (presença de microaneurismas e/ou hemorragias intraretinianas leves em 4 quadrantes e/ou veias em rosário em 1 quadrante e/ou IRMA leves em 1 a 4 quadrantes) ou grave (presença de microaneurismas e/ou hemorragias intra-retinianas graves em 4 quadrantes e/ou veias em rosário em 2 quadrantes e/ou IRMA moderadas em 1 ou mais quadrantes) e forma proliferativa (RDP) inicial (presença de neovascularização retiniana que não preenche critérios de alto risco), alto risco (NVD $<1 / 4$ dd associada à hemorragia vítrea ou qualquer NVD $>1 / 4$ dd ou NVE $>1 / 2$ dd associada à hemorragia vítrea) ou avançada (presença de descolamento de retina e/ou hemorragia vítrea densa), bem como a presença de edema macular clinicamente significativo $^{(10)}$.

Os pacientes foram divididos em dois grupos quanto à origem: grupo I (1.975 pacientes) residentes em Recife e região metropolitana; grupo II (226 pacientes) residentes no interior do estado de Pernambuco.

$\mathrm{Na}$ análise estatística, foi utilizado o teste do qui-quadrado para a comparação entre as frequiências de variáveis categóricas. Para a comparação entre variáveis contínuas foi utilizado o teste $t$ de Student. Utilizou-se $p<0,05$ para rejeição de hipótese de nulidade.

O Projeto teve início após aprovação pelo Comitê de Ética em Pesquisa da Fundação Altino Ventura.

\section{RESULTADOS}

Em relação à frequiência da retinopatia diabética, entre os pacientes do grupo I, 477 (24,2\%) diabéticos apresentavam esta complicação, enquanto que no grupo II encontrou-se em $89(39,4 \%)$ pacientes. A freqüência de RDNP para o grupo I foi de $427(21,6 \%)$ pacientes e para o grupo II, $65(28,8 \%)$ pacientes. Em relação à RDP, encontraram-se $50(2,4 \%)$ pacientes no grupo I e $24(10,6 \%)$ pacientes no grupo II (Tabela 1$).$

As frequiências do estadiamento da retinopatia diabética nos grupos estudados estão demonstradas no gráfico 1 .

$\mathrm{O}$ descolamento tracional da retina foi encontrado em 24 $(1,2 \%)$ pacientes do grupo I e em $9(4,0 \%)$ do grupo II. No grupo I, $16(0,8 \%)$ pacientes apresentavam hemorragia vítrea e no grupo II, 15 (6,6\%) pacientes. Encontrou-se edema macular clinicamente significativo em 44 (2,2\%) pacientes do grupo I e $22(9,7 \%)$ pacientes do grupo II (Tabela 2$)$.

A duração média do diagnóstico do diabetes entre os pacientes do grupo $\mathrm{I}(\mathrm{N}=1813)$ foi de 7,56 $\pm 6,92$ anos e para o

\begin{tabular}{|c|c|c|c|}
\hline Prevalência & Grupo I (\%) & Grupo II (\%) & Valor $p$ \\
\hline \multicolumn{4}{|l|}{ Forma da RD } \\
\hline Presença de RD & $477(24,2)$ & $89(39,4)$ & $<0,0001$ \\
\hline RDNP & $427(21,6)$ & $65(28,8)$ & 0,0184 \\
\hline RDP & $50 \quad(2,4)$ & $24(10,6)$ & $<0,0001$ \\
\hline
\end{tabular}

$\mathrm{RD}=$ retinopatia diabética; $\mathrm{RDNP}=$ retinopatia diabética não-proliferativa; $\mathrm{RDP}=$ retinopatia diabética proliferativa

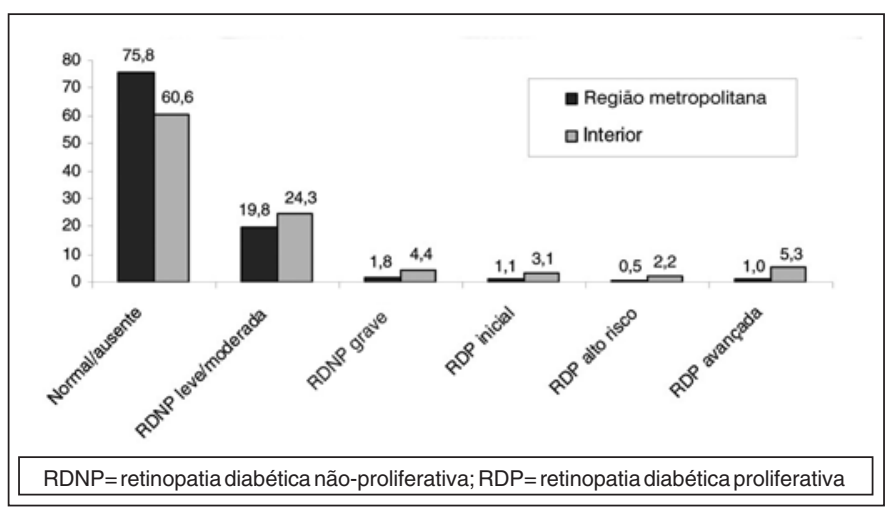

Gráfico 1 - Estadiamento da retinopatia diabética nos grupos estudados

\begin{tabular}{|c|c|c|c|}
\hline Alteração clínica & Grupo I (\%) & Grupo II (\%) & Valor $p$ \\
\hline EMCS & $44(2,2)$ & $22(9,7)$ & $<0,0001$ \\
\hline HV & $16(0,8)$ & $15(6,6)$ & $<0,0001$ \\
\hline DR & $24(1,2)$ & $9(4,0)$ & 0,0031 \\
\hline
\end{tabular}


grupo II $(\mathrm{N}=187)$ foi de $8,68 \pm 6,84$ anos $(\mathrm{p}=0,021)$. Em 201 pacientes, não foi possível a coleta deste dado por desconhecimento do tempo de diagnóstico do diabetes.

No grupo I, 427 (24,6\%) pacientes informavam já ter se submetido anteriormente a procedimento de fundoscopia e no grupo II, $46(26,6 \%)$ pacientes $(\mathrm{p}=0,6325)$.

$\mathrm{O}$ uso de insulina para controle do diabetes foi observado entre $275(14,4 \%)$ pacientes do grupo I e $41(20,6 \%)$ pacientes do grupo II. Essa diferença foi estatisticamente significante $(\mathrm{p}=0,0245)$.

\section{DISCUSSÃO}

A prevalência da RD encontrada nesse estudo para pacientes da região metropolitana de Recife foi semelhante à encontrada em outros estudos com critérios de triagem semelhantes ${ }^{(11-12)}$. Em pacientes examinados num programa de atendimento multidisciplinar do Hospital das Clínicas de Ribeirão Preto, a prevalência de RD foi de $24 \%$, incluindo diabéticos tipo I e tipo II $^{(12)}$. Ao avaliar-se a prevalência de RD em uma associação de diabéticos encontrou-se $28,2 \%$ pacientes com retinopatia ${ }^{(11)}$. Entretanto, a prevalência de RD, edema macular, hemorragia vítrea e DR tracional entre pacientes do interior, avaliados neste estudo, foi mais alta que pacientes da região metropolitana.

No Brasil, o nível de informação da população diabética em relação à possibilidade de desenvolvimento da retinopatia $\mathrm{e}$ da própria cegueira ainda é insatisfatório mesmo em pacientes que freqüentam associações destinadas a atender este tipo de paciente, havendo baixa cobertura em termos de prevenção e detecção da doença ${ }^{(11)}$.

Pacientes oriundos do interior do estado de Pernambuco encontram-se freqüentemente residindo em áreas rurais onde existe dificuldade de transporte ao centro especializado de retina que fará a triagem oftalmológica. Além disso, a divulgação da necessidade de exame fundoscópico anual e conscientização das complicações do diabetes com possibilidade de cegueira estaria ainda mais dificultado. Logo, pacientes do interior estariam sujeitos a uma forma de investigação realizada de maneira individual e procura pelo serviço de saúde de forma espontânea $^{(13)}$. Essa forma de triagem apresenta baixa cobertura populacional devido a dificuldades de acesso ao serviço de saúde e desinformação do diabético em relação à retinopatia ${ }^{(14)}$.

Os pacientes usuários de insulina desse estudo podem representar diabéticos tipo I, onde a prevalência de RD em 15 anos é de $99 \%{ }^{(15)}$ ou diabéticos tipo II que não conseguiram controle da glicose com hipoglicemiantes orais (insulino-requerentes). A maior proporção de pacientes do interior atendidos no projeto usuários de insulina e com maior tempo de diagnóstico do diabetes sugere que este grupo de pacientes está procurando atendimento oftalmológico tardiamente e, portanto mais sujeitos às complicações da doença.

Pacientes diabéticos desinformados sobre a necessidade de exame fundoscópico anual tenderiam a procurar atendimen- to oftalmológico primariamente ao aparecer sintomas visuais. Ou seja, muitas vezes quando a retinopatia já está instalada e em estágio mais adiantados, como DR tracional e hemorragia vítrea. Neste estudo foi observado que a prevalência desses estágios foi mais freqüente entre pacientes do interior. A falta de informação sobre a doença, dificuldade para transporte até o local onde está sendo realizado o projeto e ausência de unidades descentralizadas capazes de oferecer tratamento especializado poderiam ser causas dos achados encontrados nesse estudo.

Segundo a Associação Americana de Diabetes, os pacientes com diabetes mellitus insulino-dependente deveriam realizar o exame oftalmológico de 3 a 5 anos após o diagnóstico, e nos casos de diabetes mellitus não insulino-dependente no momento do diagnóstico e a partir de então, anualmente ${ }^{(16)}$. Neste estudo observou-se que apenas $24,6 \%$ dos pacientes do grupo I e 26,6\% dos pacientes do grupo II referiam ter se submetido a exame com dilatação pupilar anteriormente apesar do tempo médio de diagnóstico da doença de 7,56 (grupo I) e 8,68 (grupo II) anos. Isso mostra que a conscientização da doença em relação à necessidade de exame fundoscópico ainda não é satisfatória, havendo necessidade de medidas para a proteção dos diabéticos.

Uma das limitações desse estudo foi a ausência de dados relativos a dosagens laboratoriais como nível sérico de glicose e hemoglobina glicosilada, colesterol, avaliação da função renal, entre outros, que poderiam estar influenciando os resultados encontrados.

A implantação da telemedicina poderia ser uma alternativa para melhorar a qualidade de triagem em pacientes diabéticos residentes no interior do estado. Essa forma de triagem baseiase na transmissão de dados para centros especializados onde seria realizada a análise de exames. Constitui-se método ideal para regiões carentes de oftalmologistas e áreas geográficas de difícil acesso ${ }^{(7)}$.

A implantação de sub-sedes de prestadores de serviço e unidades descentralizadas, mais próximas da população-alvo do projeto, também poderia se constituir numa alternativa para melhoria da qualidade de atendimento e triagem de pacientes diabéticos residentes no interior do estado.

\section{ABSTRACT}

Purpose: To evaluate the prevalence and severity of diabetic retinopathy among patients cared for in a screening program in Pernambuco, Brazil, comparing regional differences between urban and rural zones. Methods: The charts of 2,223 diabetic patients (1,568 females and 655 males; mean age 59.3 \pm 12.0 years; mean duration of diabetes $8.1 \pm 6.3$ years) that took part in a screening program for diabetic retinopathy at Altino Ventura Foundation from June 2004 to June 2005 were reviewed for the presence of the disease. Patients were divided into two groups: group I, patients living in Recife and the metropolitan area; group II, patients living in the interior of 
Pernambuco state. Results: In group I, 477 (24.2\%) patients had diabetic retinophathy, while in group II, 89 (39.4\%) patients $(p<0.0001)$. The frequency of proliferative diabetic retinophathy, macular edema, vitreous hemorrhage and retinal detachment was higher in group II patients $(p<0.05)$. Conclusions: Patients living in the interior of Pernambuco state have a higher incidence of diabetic retinophathy and the advanced forms than patients living in Recife and the metropolitan area when examined in a screening program for diabetic retinopathy at the Altino Ventura Foundation. Telemedicine and descentralization actions are recommended for improvement of screening quality in patients living in the interior of Pernambuco state.

Keywords: Diabetic retinopathy/etiology; Diabetic retinopathy/diagnosis; Prevalence; Triage

\section{REFERÊNCIAS}

1. Brasil. Ministério da Saúde. Diabetes melito. Guia básico para diagnóstico e tratamento. Brasília: Sociedade Brasileira de Diabetes; 1996. p.5, 7, 57. [Programa Harvard/ Joslin/ SBD].

2. Klein R, Klein BE, Moss SE. Visual impairment in diabetes. Ophthalmology. 1984;91(1):1-9.

3. Sánchez-Thorin JC. The epidemiology of diabetes mellitus and diabetic retinopathy. Int Ophthalmol Clin. 1998;38(2):11-8. Review.

4. Sugano DM, Serra DC, Barros RA, Rehder JRCL. Impacto da retinopatia diabética em campanha de prevençäo da cegueira. Arq Méd ABC. 2001;24(1):47-50.

5. Chew EY, Klein ML, Ferris FL $3^{\text {rd }}$, Remaley NA, Murphy RP, Chantry K, et al. Association of elevated serum lipid levels with retinal hard exudate in diabetic retinopathy. Early Treatment Diabetic Retinopathy Study (ETDRS) Report 22. Arch Ophthalmol. 1996;114(9):1079-84.

6. Escarião PHG, Brandt CT, Leão ACS, Diniz JRP. Retinopatia diabética: estudo populacional de custo-benefício. An Fac Med Univ Fed Pernamb. 2003; 48(1):43-8.

7. Stefánsson E, Bek T, Porta M, Larsen N, Kristinsson JK, Agardh E. Screening and prevention of diabetic blindness. Acta Ophthalmol Scand. 2000;78(4): 374-85. Review.

8. Garvican L, Clowes J, Gillow T. Preservation of sight in diabetes: developing a national risk reduction programme. Diabet Med. 2000;17(9):627-34.

9. Early photocoagulation for diabetic retinopathy. ETDRS report number 9 . Early Treatment Diabetic Retinopathy Study Research Group. Ophthalmology. 1991;98(5 Suppl):766-85.

10. Wilkinson CP, Ferris FL $3^{\text {rd }}$, Klein RE, Lee PP, Agardh CD, Davis M, Dills D, Kampik A, Pararajasegaram R, Verdaguer JT; Global Diabetic Retinopathy Project Group. Proposed international clinical diabetic retinopathy and diabetic macular edema disease severity scales. Ophthalmology. 2003;110(9): 1677-82. Comment in: Ophthalmology. 2003;110(9):1675-6.

11. Ramos SR, Sabbag FP, Busato D, Miranda AB, Moreira Júnior CA. Retinopatia diabética: estudo de uma associação de diabéticos. Arq Bras Oftalmol. 1999;62(6):735-7.

12. Foss MC, Paccola GMGF, Souza NV, Iazigi N. Estudo analítico de uma amostra populacional de diabéticos tipo II da região de Ribeirão Preto (SP). AMB Rev Assoc Med Bras. 1989;35(5):179-83.

13. Engelgau MM, Narayan KM, Herman WH. Screening for type 2 diabetes. Diabetes Care. 2000;23(10):1563-80. Erratum in: Diabetes Care. 2000; 23(12):1868-9.

14. Hirata CE, Fang T, Casella AMB, Eliezer M, Abujamra S. Prevalência de retinopatia em uma população de diabéticos. Arq Bras Oftalmol. 1986;49(2):31-3.

15. Indications for photocoagulation treatment of diabetic retinopathy: Diabetic Retinopathy Study Report $\mathrm{n}^{\circ}$ 14. The Diabetic Retinopathy Study Research Group. Int Ophthalmol Clin. 1987;27(4):239-53.

16. Expert Committee on the Diagnosis and Classification of Diabetes Mellitus. Report of the Expert Committee on the Diagnosis and Classification of Diabetes Mellitus. Diabetes Care. 2000;23 Suppl 1:S4-19. 